\title{
IMRT Inverse Planning using Simulated Annealing
}

\author{
J. Dias ${ }^{1}$, H. Rocha ${ }^{2}$, B. Ferreira ${ }^{3,4}$ and M. C. Lopes ${ }^{3,4}$ \\ ${ }^{1}$ Faculty of Economics and Inesc-Coimbra, University of Coimbra, Portugal \\ ${ }^{2}$ Inesc-Coimbra, Rua Antero de Quental, no 199, 3000-033 Coimbra, Portugal \\ ${ }^{3}$ IPOC-FG, EPE, Av. Bissaya Barreto 98, 3000-075 Coimbra, Portugal \\ ${ }^{4}$ I3N, Campus Universitário de Santiago, 3810-193 Aveiro, Portugal
}

\begin{abstract}
Radiation therapy is an important type of cancer treatment, being delivered to more than half of all cancer patients. Intensity Modulated Radiation Therapy (IMRT) is one type of radiation therapy where it is possible not only to conform the dose distribution to the shape of the volumes to treat, but also to have different radiation intensity profiles that will allow a better coverage of the volumes to treat and a better sparing of all other structures. Planning an IMRT treatment is a time-demanding task, based on a trial and error procedure. We propose an inverse planning approach to this problem based on simulated annealing (SA) and a dynamic neighborhood concept. The planning of an IMRT treatment can be interpreted as having several stages, from choosing proper incidence directions until deciding on the movement of the multiple collimator leaves that will produce the desired intensity profiles. We consider here mainly the first of these stages: the problem of choosing the best intensity directions. This problem is known to be highly non-convex and with many local minima. SA is a well known metaheuristic that has proven to be capable of escaping local minima. Some preliminary computational results using ten clinical examples of already treated patient cases of head-and-neck tumors at the Portuguese Institute of Oncology of Coimbra (IPOC) will be described.
\end{abstract}

Keywords - Radiotherapy, IMRT, Beam Angle Optimization, Simulated Annealing, Inverse Planning.

\section{INTRODUCTION}

Radiation therapy is one type of cancer treatment that is delivered to more than half of all cancer patients. The goal of radiation therapy is to deliver a dose of radiation to the cancerous region to sterilize the tumor (radiation will damage cancer cells that will have difficulties to recover and/or will die) but at the same time minimizing the damages to the surrounding healthy organs and tissues. Typically, high energy photon beam radiation is generated by a linear accelerator mounted on a gantry that can rotate around a central axis parallel to the couch where the patient lays. The rotation of the couch combined with the rotation of the gantry allow an irradiation from almost any angle around the tumor. IMRT is one technique of radiation therapy, where the modulation of the radiation intensities is enabled by the controlled movement of the multiple leaves of the collima- tor. In conceptual terms, we can interpret a radiation beam as being composed by a grid of smaller beamlets of independent intensities. The planning of a given IMRT treatment is based on the patient's CT images and on the medical prescription. In the $\mathrm{CT}$ images, the medical doctor delineates the area(s) to treat and also all important structures that should be spared. The planner will then try to comply with the radiation oncologist treatment objectives. These constitute a list of dose prescriptions to the target volume(s) (PTVs or CTVs) and of tolerance dose-volume relationships for the organs at risk (OARs). In clinical practice, most of the times, treatment planning is done using a trial and error approach: the planner tries different sets of objectives and penalties until a satisfactory dose distribution is obtained. This is a lengthy procedure, giving no guarantees that the best possible solution is reached. A more efficient way of tackling this problem is an approach where the best set of treatment parameters complying with the medical prescription are generated by using models and optimization algorithms, with the least human interaction possible.

IMRT treatment planning can be thought as being composed of three sequential and interrelated stages: a. deciding the number of beam angles and their directions (BAO Beam Angle Optimization), where different treatment modalities can be considered (step-and-shoot, dynamic IMRT or Arc Therapy); b. calculating the optimal radiation intensity map to be delivered to the patient from every beam direction (FMO - Fluence Map Optimization); c. determining the movement of the multileaf collimator's leaves to deliver the optimal beamlet intensities (Leave Sequencing Problem). We will consider the two first planning stages, and BAO in particular, in step-and-shoot coplanar IMRT. $\mathrm{BAO}$ problem is known to be a challenging problem, highly non-convex and with many local minima. Several approaches have been proposed to tackle this problem [1-8]. SA is a well known metaheuristic that has proven to be able of calculating good quality solutions for challenging problems. In the next section we will describe the BAO inverse planning problem. In section 3 we will briefly describe the SA algorithm. Section 4 will show some preliminary computational results. Section 5 will state some conclusions and possible developments. 


\section{BAO INVERSE PLANNING PROBLEM}

In the first stage of the planning process it is necessary to determine the irradiation angles. This is usually done sequentially trying different sets of directions. Usually the number $k$ of angles to use is considered fixed. So, a given solution will be any set of $k$ angles chosen from the interval $[0,360]$ that will satisfy as much as possible the medical prescription (the medical prescription is not always attainable and assessing the quality of a given solution is a nontrivial problem). In order to apply an inverse planning approach, one has to somehow quantify the quality of each solution. This can only be done after calculating the fluence intensities to be delivered from each of the chosen angles (FMO). There are many different ways of solving the FMO problem and it is beyond the scope of this paper to discuss the appropriateness of the different approaches. Here a convex penalty function voxel-based nonlinear model [8] is used, where each voxel is penalized considering the square difference of the amount of dose received by the voxel and the amount of dose desired/allowed for the voxel. We need a way to calculate accurately the radiation dose distribution deposited in the patient. Each structure is discretized in voxels (small volume elements) and the dose is computed for each voxel using the superposition principle, i.e., considering the contribution of each beamlet. Typically, a dose matrix $D$ is such that each row of $D$ corresponds to a voxel ( $V$ in total) and each column to each possible beamlet from all beam directions considered ( $N$ in total). The element $(i, j)$ of $D$ corresponds to the dose contribution to voxel $i$ from beamlet $j$ with unit intensity. Therefore, the total dose received by the voxel $i$ is given by $\sum_{j=1}^{N} D_{i j} w_{j}$, with $w_{j}$ representing the intensity (or fluence) of beamlet $j$. The FMO can then be defined as:

$\operatorname{Min}_{w} \sum_{i=1}^{V}\left[\underline{\lambda}_{i}\left(T_{i}-\sum_{j=1}^{N} D_{i j} w_{j}\right)_{+}^{2}+\bar{\lambda}_{i}\left(\sum_{j=1}^{N} D_{i j} w_{j}-T_{i}\right)_{+}^{2}\right]$

s.t. $w_{j} \geq 0, j=1, \cdots, N$

where $T_{i}$ is the desired dose for voxel $i, \underline{\lambda}_{i}$ and $\bar{\lambda}_{i}$ are the penalty weights of underdose and overdose of voxel $i$, respectively, and $(\cdot)_{+}=\max \{0, \bullet\}$. This nonlinear formulation implies that a very small amount of underdose or overdose may be accepted in clinical decision making, but larger deviations from the desired/allowed doses are decreasingly tolerated. If we define $\Theta$ as the set of all possible angles, then $\mathrm{BAO}$ problem can be defined as follows: $\min f\left(\theta_{1}, \theta_{2}, \cdots, \theta_{k}\right)$

subject to $\theta_{1}, \cdots, \theta_{k} \in \Theta$

where $f(\boldsymbol{\theta}), \boldsymbol{\theta}=\theta_{1}, \cdots, \theta_{k} \in \Theta$, is given by (1) considering all beamlets belonging to $\boldsymbol{\theta}$ and only these. The aim is to optimize (2) in an automated way, without human intervention, by applying a SA algorithm.

\section{SA ALGORITHM}

SA can be interpreted as a local search probabilistic procedure that will try to find a global minimum of a cost function even in the presence of many local minima [9]. SA is inspired by the thermal process for obtaining low energy states of a solid in a heat bath in condensed matter physics [10]. Detailed descriptions of SA can be found in [9-12]. Given a set $S$ of possible solutions to the problem, a function $f$ that will allow us to compare two solutions, a neighborhood structure and a non-increasing function $T: N \rightarrow(0, \infty)$, SA algorithm can be described as follows:

1. Set counter $i \leftarrow 1$; Define the initial solution $x \_$current and calculate $f \_$current.f_best $\leftarrow f$ _current $; x \_$best $\leftarrow x$ _current .

2. Generate randomly a new solution ( $x_{-}$new $)$in the neighborhood of $x \_$current.$x_{-}$current $\leftarrow x \_n e w$. Evalute this solution $\left(f_{-}\right.$current $)$.

3. If $f_{-}$current $\leq f \_$best then $f_{-}$best $\leftarrow f_{-}$current and $x \_$best $\leftarrow x \_$current. Go to 5 .

4. Calculate $a u x \leftarrow e^{-\frac{\left(f_{-} \text {current }-f_{-} \text {best }\right)}{T(i) \times f_{-} \text {best }}}$. Generate a random number $n \in[0,1]$ from a uniform distribution. If $n>a u x$ then $f_{-}$current $\leftarrow f_{-}$best and $x \_$current $\leftarrow$ _ $\_$best.

5. $i \leftarrow i+1$. If the stopping criterion is not met go to 2. Else stop.

Step 1 initializes the procedure. In Step 2 a solution is randomly selected from the neighborhood of the current solution. If this new generated solution is better than the best one known so far, it will become the best solution and the best known value of the objective function is updated (Step 3). If this new solution is worse than the best solution, it can still be considered the current one in the next iteration with a given probability (Step 4). Otherwise, the current solution in the next iteration will be the best one known so far. This probability will depend on the current value of function $T(T(i))$. The procedure is repeated until some stopping criterion is met (in the present case a maximum number of iterations). We have defined the initial solution to be the one with equidistant angles, since this is usually the solution used in clinical practice. The quality of each solution is given by (1). Two solutions are considered to be neighbors if they differ by at most $z$ angles. The value of $z$ is dynamically and stochastically determined in each iteration by using the concept of dynamically dimensioned 
search spaces [13]. Each variable (angle) will be perturbed with a given probability calculated as $1-\log (i) / \log (N)$ where $N$ is the maximum number of iterations considered. This means that larger neighborhoods are considered in the beginning of the algorithm's execution, promoting a more global search. The algorithm will then become focused in the small neighborhood of the current solution. The magnitudes of the perturbations are randomly sampled from a normal distribution with mean 0 and standard deviation $r$ (considered equal to $360 / 2 k$ ). Given the specificities of BAO problem, it is also guaranteed that the current solution does not have two adjacent angles that are less than $4^{\circ}$ apart (considered the same from a clinical point of view). Function $T$ (also known as temperature or cooling schedule due to the analogy with the physical annealing procedure) is here defined as $T(i)=(1-\log (i) / \log (N))$. The probability of accepting a worse solution than the current one is greater at the beginning of the algorithm and is close to zero in the last iterations.

\section{Computational Results}

The SA algorithm was tested considering ten clinical cases of already treated head-and-neck cancer patients at IPOC, signalized as complex cases where proper target coverage and organ sparing proved to be difficult to obtain. The medical prescription was similar for all these patients (Table 1). There are two PTVs with different dose prescriptions. The simplified OAR list includes the spinal cord, brainstem and parotids.

Table 1 Prescribed doses for all the structures considered

\begin{tabular}{lccc}
\hline Structure & Mean dose & Maximum Dose & Prescribed Dose \\
\hline Spinal cord & - & $45 \mathrm{~Gy}$ & - \\
Brainstem & - & $54 \mathrm{~Gy}$ & - \\
Left parotid & $26 \mathrm{~Gy}$ & - & - \\
Right parotid & $26 \mathrm{~Gy}$ & - & - \\
PTV1 & - & - & $70.0 \mathrm{~Gy}$ \\
PTV2 & - & - & $59.4 \mathrm{~Gy}$ \\
Body & - & $80 \mathrm{~Gy}$ & - \\
\hline
\end{tabular}

The spinal cord and the brainstem are some of the most critical OARs in the head-and-neck tumor cases, because even if only one subunit is damaged, the whole organ functionality is compromised (serial type organ). Parotid glands are the largest of the three salivary glands and their irradiation can cause xerostomia (dry mouth due to lack of saliva), decreasing the quality of life of patients due to difficulties to swallow. The parotids are parallel type organs, i.e., if a small volume of the organ is damaged, the rest of the organ functionality may not be affected, so we should try to guar- antee a minimum mean dose in the delivered treatment. Treatments with five coplanar beams are considered, since in this case beam direction is critical/fundamental to achieve a good treatment plan. An increase in the number of angles is only considered if it is not possible to reach a clinically acceptable solution with this number of angles. Treating a patient with a reduced number of angles has several advantages for the patient, by reducing setup errors and irradiation time, and for the workflow of the health institution, allowing to increase the number of treated patients. One of the difficulties a planner faces is to know whether or not an increased number of beams should be considered, since it is not possible for him to explore the whole feasible space and to know with a high level of certainty that it is not possible to find an acceptable solution with the current number of beams. An angle optimization approach can support the decision of considering treatment plans with more angles.

Our tests were performed on a Intel Core i7 CPU 2.8 $\mathrm{GHz}$ 4GB RAM Windows 7 PC. The dose was computed by CERR's pencil beam algorithm [14] (v. 3.2.2, Matlab $\mathrm{R} 2007 \mathrm{a})$. For each of the ten cases, the voxel size was $0.3 \times 0.3 \times 0.3 \mathrm{~cm}^{3} . N$ was set to 200 . The FMO problem was solved by a trust-region-reflective algorithm (fmincon, Matlab Optimization Toolbox). Each instance of the FMO took from 56 to 350 seconds to be solved. The results consider the improvement in the objective function compared to the equidistant solution. For each patient, the algorithm was executed five times.

Table 2 Computational Results

\begin{tabular}{lrrrr}
\hline Patient & $\begin{array}{r}e q u i \\
\text { solution }\end{array}$ & $\begin{array}{r}\text { Average SA } \\
\text { Solution }\end{array}$ & $\begin{array}{r}\text { Standard } \\
\text { Deviation }\end{array}$ & $\begin{array}{r}\text { Average \% } \\
\text { improvement }\end{array}$ \\
\hline 1 & 387.3 & 374.6 & 1.4 & $3.3 \%$ \\
2 & 72.9 & 68.4 & 0.8 & $6.2 \%$ \\
3 & 187.6 & 173.6 & 2.2 & $7.5 \%$ \\
4 & 156.4 & 150.0 & 1.0 & $4.1 \%$ \\
5 & 277.6 & 256.9 & 1.5 & $7.4 \%$ \\
6 & 165.6 & 155.1 & 1.5 & $6.3 \%$ \\
7 & 40.4 & 34.0 & 0.5 & $15.8 \%$ \\
8 & 165.0 & 153.4 & 1.1 & $7.7 \%$ \\
9 & 124.3 & 116.0 & 2.3 & $6.6 \%$ \\
10 & 186.4 & 178.3 & 2.9 & $4.4 \%$ \\
\hline
\end{tabular}

More important than the value of the objective function are the results from the clinical point of view, looking at the irradiated doses in the structures considered. A metric usually used for plan evaluation is the PTV volume that receives $95 \%$ of the prescribed dose (usually $95 \%$ of the PTV volume is required). These metrics are displayed for the ten cases in Fig. 1, considering the best and the worst solutions out of the 5 solutions generated by SA and also the equidistant solution. The horizontal lines represent $95 \%$ of the prescribed dose. In order to verify parotids' sparing, mean doses are usually displayed (Fig. 2). The horizontal lines 
represent the tolerance mean dose for the corresponding structures. Satisfactory treatment plans should obtain results under these lines. The doses for spinal cord and brainstem fulfilled the maximum dose tolerance in all tested cases and for all treatment plans, so are not depicted.
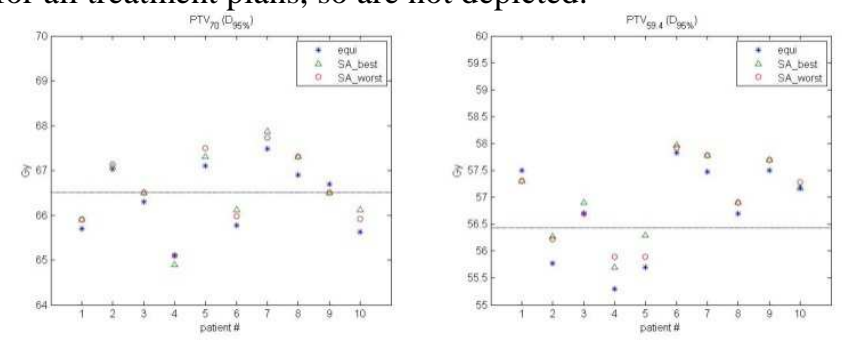

Fig. 1 Comparison of target irradiation metrics
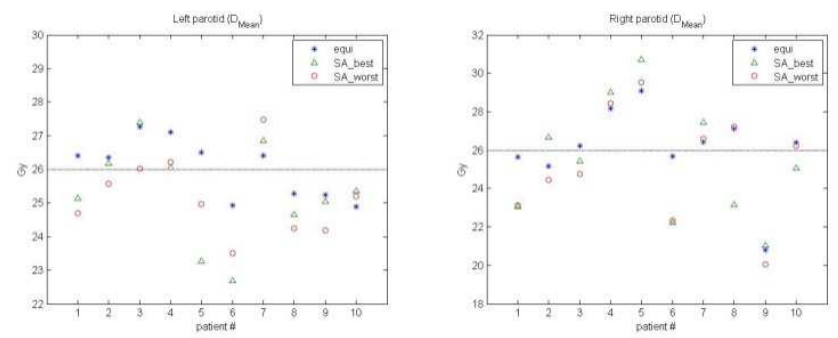

Fig. 2 Comparison of organ sparing metrics

The results show that it was possible to improve treatment plans with respect to all structures in 7 out of 10 patients. The PTV coverage has improved for all patients, and it was possible to obtain significant improvements in the left parotid sparing. In these clinical cases, the right parotid has proven to be more difficult to spare. Nevertheless, for five out of the ten patients it was necessary to increase the number of beams to seven since the dose distribution was not considered acceptable.

\section{CONCLUSIONS}

Applying SA to BAO inverse planning problem improves organ sparing without jeopardizing tumor coverage. The use of automated optimization methods in IMRT planning problems diminishes the time needed for planning, whilst at the same time improves the quality of treatments. Other algorithmic approaches capable of dealing with high non-convex search spaces with many local minima should be investigated, like tabu search, path relinking, among others. Another possibility is taken advantage of already treated patients' data and applying knowledge-based approaches.

\section{ACKNOWLEDGMENT}

This work was supported by FEDER, COMPETE, iCIS (CENTRO-07-ST24-FEDER-002003), Portuguese Foundation for Science and Technology under project grants PTDC/EIA-CCO/121450/2010, PEst-OE/EEI/UI308/2014. The work of H. Rocha was supported by the European Social Fund and Portuguese Funds.

\section{CONFLICT OF INTEREST}

The authors declare that they have no conflict of interest.

\section{REFERENCES}

1. Li, Y., J. Yao, and D. Yao, Automatic beam angle selection in IMRT planning using genetic algorithm. Physics in Medicine and Biology, 2004. 49: p. 1915.

2. Li, Y., D. Yao, J. Yao, and W. Chen, A particle swarm optimization algorithm for beam angle selection in intensity-modulated radiotherapy planning. Physics in Medicine and Biology, 2005. 50: p. 3491.

3. Craft, D., Local beam angle optimization with linear programming and gradient search. Physics in Medicine and Biology, 2007. 52(7): p. N127-N135.

4. Dias, J., H. Rocha, B. Ferreira, and M.C. Lopes, A genetic algorithm with neural network fitness function evaluation for IMRT beam angle optimization. Central European Journal of Operations Research, 2013. accepted for publication.

5. Dias, J., H. Rocha, B.C. Ferreira, and M.C. Lopes. IMRT Beam Angle Optimization Using Dynamically Dimensioned Search. in The International Conference on Health Informatics, IFMBE Proceedings 42, p. 1-4. 2013.

6. Zhang, H.H., S. Gao, W. Chen, L. Shi, W.D. D'Souza, and R.R. Meyer, A surrogate-based metaheuristic global search method for beam angle selection in radiation treatment planning. Physics in Medicine and Biology, 2013. 58(6): p. 1933-1946.

7. Rocha, H., J. Dias, B.C. Ferreira, and M.C. Lopes, Selection of intensity modulated radiation therapy treatment beam directions using radial basis functions within a pattern search methods framework. Journal of Global Optimization, 2013. 57: p. 1065-1089.

8. Aleman, D.M., A. Kumar, R.K. Ahuja, H.E. Romeijn, and J.F. Dempsey, Neighborhood search approaches to beam orientation optimization in intensity modulated radiation therapy treatment planning. Journal of Global Optimization, 2008. 42(4): p. 587-607.

9. Bertsimas, D. and J. Tsitsiklis, Simulated annealing. Statistical Science, 1993: p. 10-15.

10. Aarts, E., J. Korst, and W. Michiels, Simulated Annealing, in Search Methodologies. 2005, Springer. p. 188-210.

11. Kirkpatrick, S., D.G. Jr, and M.P. Vecchi, Optimization by simulated annealing. Science, 1983. 220(4598): p. 671-680.

12. Ingber, L., Simulated annealing: Practice versus theory. Mathematical and Computer Modelling, 1993. 18(11): p. 29-57.

13. Tolson, B. and C. Shoemaker, Dynamically dimensioned search algorithm for computationally efficient watershed model calibration. Water Resources Research, 2007. 43 (1).

14. Deasy, J.O., A.I. Blanco, and V.H. Clark, CERR: A computational environment for radiotherapy research. Medical Physics, 2003. 30: p. 979-985. 